\title{
DNA probe detection within 3D hydrogel matrix in a hollow core photonic crystal fibre
}

Rutowska, Monika, Lu, Jiadi, Garcia-Gunning, Fatima, Ellis, Andrew

Monika Rutowska, Jiadi Lu, Fatima C. Garcia-Gunning, Andrew D. Ellis, "DNA probe detection within 3D hydrogel matrix in a hollow core photonic crystal fibre," Proc. SPIE 7753, 21 st International Conference on Optical Fiber Sensors, 77536I (17 May 2011); doi: 10.1117/12.886070

SPIE Event: 21st International Conference on Optical Fibre Sensors (OFS21), 2011, Ottawa, Canada 


\title{
DNA Probe Detection within 3D Hydrogel Matrix in a Hollow Core Photonic Crystal Fibre
}

\author{
Monika Rutowska, Jiadi Lu, Fatima C. Garcia Gunning and Andrew D. Ellis \\ Photonic Systems Group \\ Tyndall National Institute and Department of Physics, University College Cork, Ireland
}

\begin{abstract}
In this paper, we report for the first time the detection of a Cy5-labelled DNA probe immobilised within a 3D hydrogel matrix, formed inside a hollow core Photonic Crystal Fibre (HC-PCF). We show both the sensitivity of fluorescence detection inside the HC-PCF using a supercontinuum light source and of the variation of the luminescence intensity with the different concentrations of DNA probe within the hydrogel. The 3D hydrogel matrix is a network of polymer chains, which is expected to provide highly sensitive detection and selection of bio-molecules, in comparison with 2D coverage. The biocompatibility of hydrogel in the HC-PCF suggests numerous applications associated with immobilised DNA probe detection for point-of-care or remote systems.
\end{abstract}

Keywords: Photonic crystal fibres, hollow core, biosensing, silanisation, hydrogel, DNA, fluorescence

\section{INTRODUCTION}

The use of microstructure fibres for numerous sensing applications is rapidly expanding. The detection of molecules in gas or liquids, as well as the detection of bio-molecules anchored inside the fibres have established MOFs as sensitive and reliable candidates for sensors ${ }^{1-4}$. Such fibres are unique, due to the light guiding mechanism inside their structure which enables to shift the guided frequencies by changing the refractive index within their holes ${ }^{6}$ and improves the light interaction within sample.

In a recent paper, we propose to combine the optical benefits of such fibres, especially the HC-PCF, and use them for molecule immobilisation, tagging, and measurement based on hydrogel ${ }^{7}$. Hydrogel is a competing technology which offers three dimensional matrix for the immobilisation of molecules and it is considered to offer higher sensitivity than two dimensional immobilisation, common to a surface coverage devices ${ }^{8}$ for the detection and selection of attached biomolecules inside its structure. It consists a network of polymer chains cross-linked via chemical bonds. Hydrogel has been widely used for biomedical applications ${ }^{9}$ due to its hydrophilic character and biocompatibility ${ }^{10}$, which are mainly due to the well controlled growing process of hydrogel, swelling, molecular diffusion and permeability ${ }^{10-12}$.

Individually, both of these approaches offer advantages over conventional micro-array assays, and would offer significant benefits for DNA based research for a variety of health applications. In this paper, we combine the 3D immobilisation properties of hydrogel with the improved ligand-light interaction of HC-PCFs, reporting for the first time, the detection of the immobilised DNA probe inside a 3D hydrogel (25\% PEG-DA w/v) structure formed within a HCPCF, and present the sensitivity of the system for fluorescence measurements, using a supercontinuum source. In this system, fluorescence tagged DNA is bound to pre-polymer molecules, which are injected into the HC-PCF. Hydrogel formation is then photo-initiated enabling subsequent fluorescence analysis by illuminating the fibre at the absorption wavelength of the flourochrome. The HC-PCF enables an interaction length of several $\mathrm{cm}$ and provides an efficient collection mechanism for the emitted fluorescence.

\section{HC-PCFs PREPARATION}

To form hydrogel within the HC-PCF, that will enable the DNA probe detection, chemical surface preparation, treatment and photo-polymerisation process are required inside the fibre. The photo-polymerisation process occurs in the frequency range of the shifted bandgap, which is $510-680 \mathrm{~nm}$. In a HC-PCF $1060^{6}$ with a core diameter of $9.8 \mu \mathrm{m}$, this is achieved by changing the refractive index to 1.33 . Therefore, the first batch of HC-PCFs 1060 was silanised following a specific chemical protocol as described in $^{7}$. Briefly, the internal surface of each fibre was covered with silane agents

21st International Conference on Optical Fiber Sensors, edited by Wojtek J. Bock, Jacques Albert, Xiaoyi Bao, Proc. of SPIE Vol. 7753, 77536I · @ 2011 SPIE · CCC code: 0277-786X/11/\$18 · doi: 10.1117/12.886070 
(solution of AMPTS 3\%) and photo-initiator molecules (solution of $0.5 \mathrm{mM}$ Eosin Y and $25 \mathrm{mM}$ EDAC) resulting in fibre cross sections under white light illumination, as shown in Fig. 1.

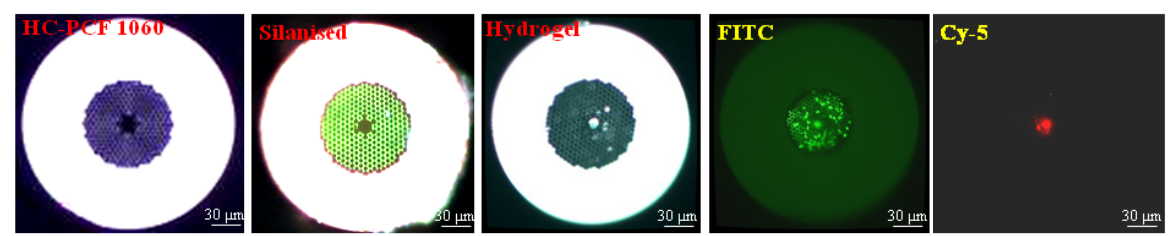

Fig. 1. The images of HC-PCFs 1060 , where from the left side: $\left(1^{\text {st }}\right)$ HC-PCF $1060,\left(2^{\text {nd }}\right)$ after the silanisation process, $\left(3^{\text {rd }}\right)$ the hydrogel formed in the core, $\left(4^{\text {th }}\right)$ the FITC image for a hydrogel, $\left(5^{\text {th }}\right)$ the Cy-5 DNA immobilisation in the fibre core.

As detailed in ${ }^{7}$, a 25\% PEG-DA pre-polymer was prepared also here, which was a phosphate buffer (PBS), consisting of $25 \%$ (v/v) poly(ethylene glycol) diacrylate (PEG-DA), $225 \mathrm{mM}$ triethanolamine (TEA), and $37 \mathrm{mM}$ 1-vinylpyrollidinone (VP) which could be polymerised into hydrogel by photo-initiation. A $100 \mu \mathrm{M}$ concentration of amino-modified oligonucleotides (Metabion International AG), with attached Cy-5 Fluor, were added to pre-polymer with a NHS-PEGacrylate cross-linker. The pre-polymer solutions were injected into fibre samples using a syringe pump setup and uniform hydrogel growth in the core was initiated by focussing a $50 \mathrm{~mW} 532 \mathrm{~nm}$ laser into the fibres samples using a x4 lens. The light was transmitted through the fibre samples for approximately 15 minutes.

To investigate the sensitivity of the optical system for use in the latter phase of experiments, another fibre batch of HCPCFs 1060 was also prepared. After changing the refractive index of the fibre core to 1.33 , we enabled guiding the wavelength range of $650-750 \mathrm{~nm}$ and not of the original wavelength at $1060 \mathrm{~nm}$. Since the majority of commercially available fluorochromes, suitable for DNA tagging, interact in or near the visible wavelength range and are water soluble compounds, we decided to apply selective filling, with the help of the fusion splicing method ${ }^{13}$. Hence we cleaved HCPCFs 1060 into pieces of approximately $30 \mathrm{~cm}$ in length and modified the front-end of each one of them up to a structure depth of $100 \mu \mathrm{m}$. Then we were able to fill only the core of the prepared fibre pieces with fluorochrome sample, diluted in water.

\section{EXPERIMENTS AND RESULTS}

The optical setup for the DNA probe detection is shown in Fig. 2. The source is a supercontinuum ${ }^{14}$ generated by a QSwitched semiconductor pumped Nd:YAG laser, centred at $1064 \mathrm{~nm}(6.85 \mathrm{kHz}$ repetition rate, $0.55 \mathrm{~ns}$ pulse width, $\sim 10.7 \mu \mathrm{J}$ pulse energy) together with a $20 \mathrm{~m}$ highly nonlinear PCF (NL-PCF), covering the range of wavelengths from $350 \mathrm{~nm}$ to $1700 \mathrm{~nm}$. The light was collimated with an infinity corrected objective, and passed through a series of filters. To obtain the optimum excitation of the fluorochrome and detection of the resulting a back-fluorescence, selected filters were placed before and after the sample, as detailed in Table 1. The fluorescence was collected through a focusing lens (x10), coupled into a multimode fibre which was connected to a photomultiplier tube with a sensitivity peak at $630 \mathrm{~nm}$. A 16 bit 400ks/s analogue to digital converter was used to record data, which was then processed to give the total number of fluorescence counts in a 0.1 second interval.

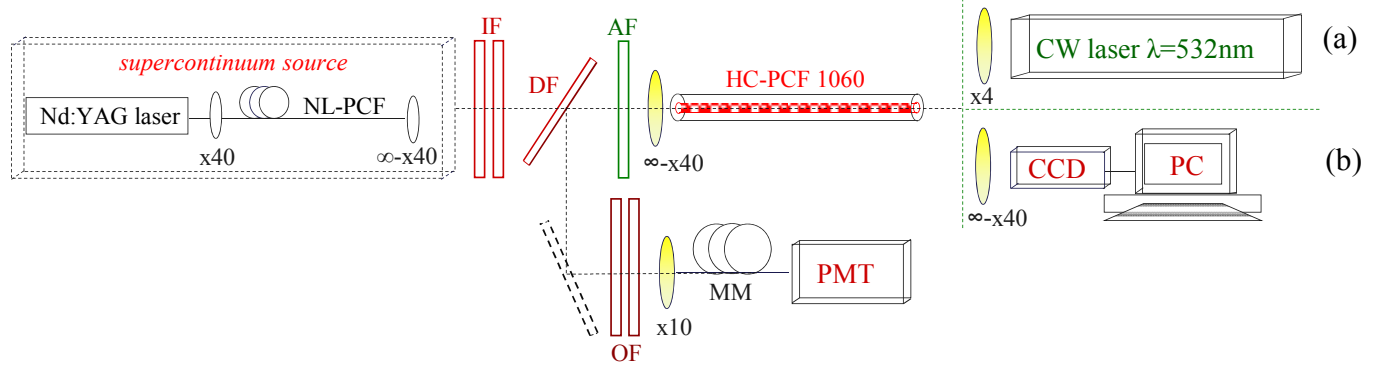

Fig. 2: Optical experimental setup where: $\mathrm{NL}-\mathrm{PCF}=$ highly nonlinear $\mathrm{PCF}, \mathrm{IF}=$ input filters, $\mathrm{DF}=$ dichroic filter, $\mathrm{AF}=\mathrm{alignment}$ filter, $\mathrm{OF}=$ output filters, $\mathrm{PMT}=$ Photomultiplier tube, $\mathrm{MM}=$ multi-mode fibre. The HC-PCF output was either connected to (a) a $\mathrm{CW}$ laser for the photo-initiation, or (b) a CCD for monitoring purposes. 


\begin{tabular}{|c|c|c|c|c|}
\hline Filters & \multicolumn{2}{|l|}{ Cy-5 Fluor } & \multicolumn{2}{|l|}{ Alexa 750 Fluor } \\
\hline Input Filters & band pass filter at $650 \mathrm{~nm}, 10 \mathrm{~nm}$ bandwidth & low pas s filter, at $650 \mathrm{~nm}$ & band pass filter at $740 \mathrm{~nm}, 10 \mathrm{~nm}$ bandwidth & low pass filter, at $750 \mathrm{~nm}$ \\
\hline Output Filters & band pass filter at $670 \mathrm{~nm}, 10 \mathrm{~nm}$ bandwidth & high pas s filter, at $665 \mathrm{~nm}$ & band pass filter at $780 \mathrm{~nm}, 10 \mathrm{~nm}$ bandwidth & high pass filter, at $775 \mathrm{~nm}$ \\
\hline Dichroic Filter & \multicolumn{2}{|c|}{ transmission at $650 \mathrm{~nm}$, reflection a $670 \mathrm{~nm}$} & \multicolumn{2}{|c|}{ transmission at $750 \mathrm{~nm}$, reflection a $780 \mathrm{~nm}$} \\
\hline
\end{tabular}

Table 1. The description of set filters for a Cy-5 Fluor and Alexa 750.

To verify the sensitivity of the system at the predicted loss minima of water filled HC-PCF, the prepared fibre samples were filled with the various concentrations of Alexa Fluor 750 (diluted in distilled water), from $500 \mathrm{nM}$ to $0.5 \mathrm{nM}$, with excitation and emission wavelengths of $750 \mathrm{~nm}$ and $780 \mathrm{~nm}$, respectively. The average launched power into the fibre was around $62 \mu \mathrm{W}$. The fluorescence bleaching curves, for the different concentrations, are shown in Fig. 3(a), where the lowest detectable concentration of flourochrome was $1 \mathrm{nM}$. Given a fibre length of $33 \mathrm{~cm}$ this corresponded to a $24 \mathrm{~nL}$ volume of sample $\left(\sim 14 \times 10^{6}\right.$ flourochrome molecules). To enable the detection of lower concentrations, the fusion splicing parameters were set to modify the selective filling process and the sample was allowed to fill the core and 18 additional capillaries in the cladding. As a result, a lower concentration of $0.5 \mathrm{nM}$ was detectable with the fibre length of $31 \mathrm{~cm}$, corresponding to the sample volume of $53 \mathrm{~nL}\left(\sim 16 \times 10^{6}\right.$ flourochrome molecules) as shown in Fig. 3(b).
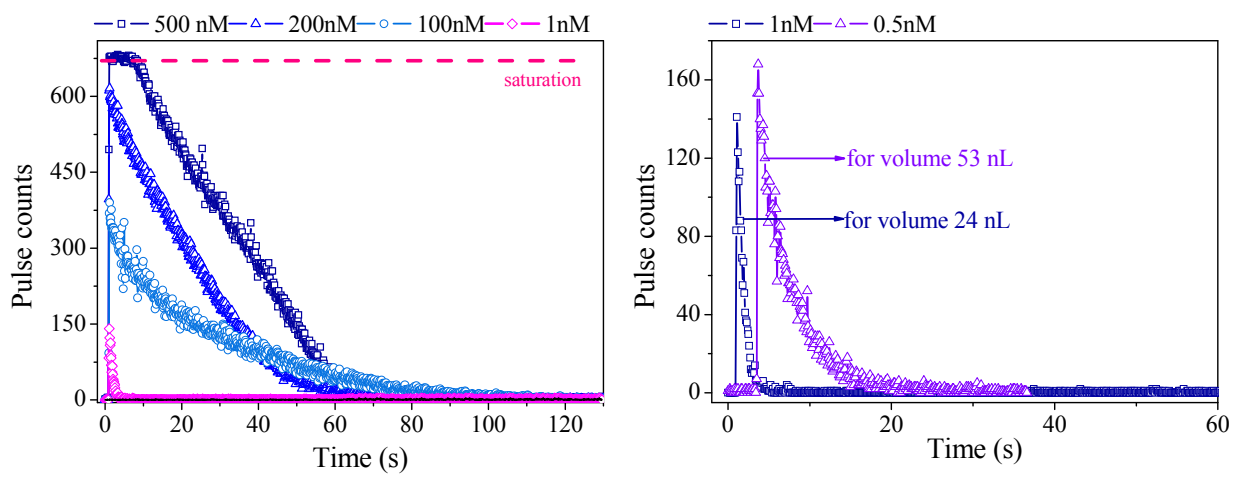

Fig. 3: (a) Fluorescence decay curves as a function of concentration of Alexa-Fluor 750, (b) Flourescence decay curves for $1 \mathrm{nM}$ filled core (square symbols) and $0.5 \mathrm{nM}$ filled core and surrounding 18 holes (triangular symbols).
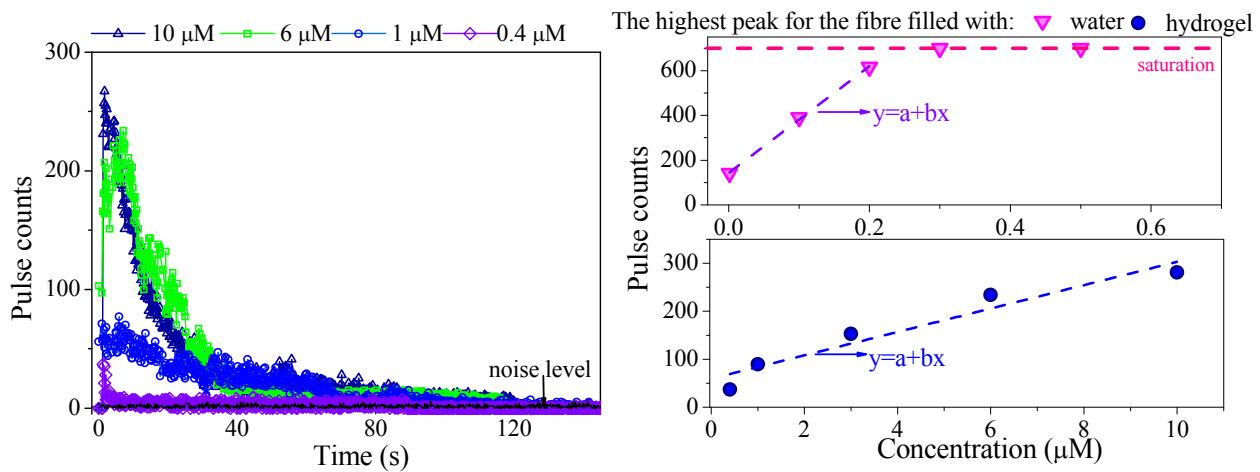

Fig. 4: (a) Fluorescence decay curves as a function of concentration for a Cy-5 DNA probe incorporated in a fibre filled with hydrogel, (b) comparison of fluorescence peak intensity for Alexa-Fluor 750 in water (top), and hydrogel immobilised oligos (bottom).

Although the refractive index of hydrogel is close to that of water, it was found that fibre filled with hydrogel guided wavelengths closer to $650 \mathrm{~nm}$ than to $750 \mathrm{~nm}^{15}$. In order to work at this shorter wavelength Cy-5 Flour was ordered to be attached to the oligos array. Cy-5 has excitation and emission wavelengths at $650 \mathrm{~nm}$ and $670 \mathrm{~nm}$, respectively. Before the fluorescence measurements, a hydrogel filled fibre was observed using the microscope to confirm confinement of the hydrogel to the fibre core, as shown in Fig. 1 ( $3^{\text {rd }}$ from the left). Fig. 1 also shows the auto-fluorescence of the hydrogel and the surface coverage due to photo-sensitive Eosin Y ( $4^{\text {th }}$ from the left), detected with a 490/520 nm dichroic filter. The Fig. 1 ( $5^{\text {th }}$ image from the left) was taken using $650 / 670 \mathrm{~nm}$ dichroic filter shows that the DNA was immobilised within hydrogel, at the core of the HC-PCF. Quantitative fluorescence measurements were then arranged using the setup of Fig. 2, with Cy-5 filters set, as detailed in Table 1. However, the average illumination, using the full set input filters, 
for wavelength of $650 \mathrm{~nm}$ was only $14 \mu \mathrm{W}$, due to the supercontinuum intensity profile. From measurements taken using the HC-PCF 1060 filled with hydrogel, we observed a higher transmission loss than for pure water measurements. Hence, we applied the average illumination of $260 \mu \mathrm{W}$, which was achieved by removing the band pass filter at $650 \mathrm{~nm}$. The output of fibre was monitored with CCD camera confirm for all measurements that the light was correctly focused into the filled core and a strong interaction between the light and the sample was achieved, at last the fluorescence decay curves were detected. Fig. 4(a) shows the corresponding fluorescence decay curves for concentrations varying from 0.4 $\mu \mathrm{M}$ to $10 \mu \mathrm{M}$, using HC-PCF 1060 lengths of approximately $10 \mathrm{~cm}$. Measurable responses were observed even for the lowest oligos concentration of $0.4 \mu \mathrm{M}\left(\sim 1.7 \times 10^{9}\right.$ oligos $)$ immobilised in the HC-PCF. Fig. 4(b) shows the variation in the fluorescence amplitude, measured at the peak of the fluorescence decay curve with sample concentration for both water with Alexa-Fluor 750 (upper curve) and hydrogel with Cy-5 DNA oligos. The data clearly demonstrates PMT saturation for the highest concentrations, however, a linear fit is observed for concentrations up to $0.2 \mu \mathrm{M}$ for the Alexa-Fluor 750 reference, and around $10 \mu \mathrm{M}$ for the Cy-5 DNA oligos fibres. A larger dynamic range would clearly be available using a set of neutral density filters.

Note that these results were obtained using a standard 25\% PEG-DA (w/v) solution. This concentration has been shown previously, as being the optimum for allowing variations in the PEG-DA, TEA, and VP chemical compounds to alter optimise the permeability of hydrogel, as well as, the crosslink density, and thickness coverage ${ }^{10-12}$. Re-optimisation of the pre-polymer solution and the optical properties of the HC-PCF might optimise guidance at the fluorescence wavelength when filled with hydrogel, and is expected to result in higher sensitivity of the immobilised probes within hydrogel in the fibre.

\section{CONCLUSION}

We have demonstrated the Cy5-labelled DNA oligonucleotides detection within a 3D hydrogel matrix formed inside the holes of a hollow-core PCF. A $0.4 \mu \mathrm{M}$ oligonucleotides sensitivity was observed via fluorescence measurements using a $260 \mu \mathrm{W}$ illumination. The potential sensitivity of the scheme is demonstrated, using water based solutions in the HCPCF 1060 , to be in the range of $0.5 \mathrm{nM}$.

\section{ACKNOWLEDGEMENTS}

The authors would like to acknowledge Dr. Andy Ruth and Dr. Jon Ellis for support and useful discussions. This material is based upon work supported by the Science Foundation Ireland under grant number 06/IN/I969.

\section{REFERENCES}

[1] Hoiby P.E. et al. "Molecular immobilization and detection in a photonic crystal fiber" Proc. SPIE, 5317, 220-23 (2004)

[2] Debs J.E. et al. "A Fundamental Study Into the Surface Functionalization of Soft Glass Microstructured Optical Fibers via Silane Coupling Agents" J. Lightwave Technol. 27(5), 576-582 (2009)

[3] Schartner E.P.et al. "Practical sensitive fluorescence sensing with microstructure fibers" Proc. SPIE 7503(5X), (2009)

[4] Huyang G. et al. "Remote Gaseous Acid Sensing within a Porphyrin-Doped TiO2 Sol-Gel Layer inside a Structured Optical Fibre" Proc. SPIE 7653(Q), (2010)

[5] Knight J.C. "Photonic crystal fibres" Nature, 424, 847-851 (2003)

[6] Antonopoulos G. et al. "Experimental demonstration of the frequency shift of bandgaps in photonic crystal fibers due to refractive index scaling" Opt. Exp.14(7), 3000-3006 (2006)

[7] Rutowska M.S. et al., "Integration of a 3D hydrogel matrix within a hollow core photonic crystal fibre for DNA probe immobilization" Meas. Sci. Technol. 21(094016), (2010)

[8] Yershov G. et al. "DNA analysis and diagnostic on oligonucloetide microchips" Proc. Natl. Acad. Sci. 93, 4913-4918 (1996)

[9] Charles P.T. et al. "Fabrication and characterization of 3D hydrogel microarrays to measure antigenicity and antibody functionality for biosensor applications", Biosen. Bioelectr. 20, 753-764 (2004)

[10] Kizilel S. et al. "Mathematical Model for Surface-Initiated Photopolymerization of Poly(ethylene glycol) Diacrylate" Macromol. Theory Simul, 15, 686-700 (2006)

[11] Ehrick J.D. et al. "Genetically engineered protein in hydrogels tailors stimuli-responsive characteristics" Nat. 4, 298-302 (2005)

[12] Kizilel S. et al. "Modelling of PEG Hydrogel Membranes for Biomedical Applications" Macromol. React. 3, 271-287 (2009)

[13] Nielsen K. et al. "Selective filling of photonic crystal fibers" J. Opt. A: Pure Appl. Opt. 7(8), L13-20 (2005)

[14] Dudley J.M. et al. "Supercontinuum generation in air-silica microstructured fibers with nanosecond and femtosecond pulse pumping” Opt. Soc. Am B, 19(4), 765-771 (2002)

[15] Rutowska M. et al. “Optical Properties of Hydrogel-Filled Hollow Core Photonic Crystal Fibres” Proc. SPIE 7653(P), (2010) 